\title{
Schistosomiasis Vaccine Development: Progress and Prospects
}

\author{
NR Bergquist \\ UNDP/World Bank/WHO Special Programme for Research and Training in Tropical Diseases (TDR), WHO, \\ CH-1211 Geneva 27, Switzerland
}

The undisputed, worldwide success of chemotherapy notwithstanding, schistosomiasis continues to defy control efforts in as much rapid reinfection demands repeated treatment, sometimes as often as once a year. There is thus a need for a complementary tool with effect for the longer term, notably a vaccine. International efforts in this direction have been ongoing for several decades but, until the recombinant DNA techniques were introduced, antigen production remained an unsurmountable bottleneck. Although animal experiments have been highly productive and are still much needed, they probably do not reflect the human situation adequately and real progress can not be expected until more is known about human immune responses to schistosome infection. It is well-known that irradiated cercariae consistently produce high levels of protection in experimental animals but, for various reasons, this proof of principle cannot be directly exploited. Research has instead been focussed on the identification and testing of specific schistosome antigens. This work has been quite successful and is already at the stage where clinical trials are called for. Preliminary results from coordinated in vitro laboratory and field epidemiological studies regarding the protective potential of several antigens support the initiation of such trials. A series of meetings, organized earlier this year in Cairo, Egypt, reviewed recent progress, selecteded suitable vaccine candidates and made firm recommendations for future action including pledging support for large-scale production according to good manufacturing practice (GMP) and Phase I trials. Scientists at the American Centers for Disease Control and Prevention $(C D C)$ have drawn up a detailed research plan. The major financial support will come from USAID, Cairo, which has established a scientific advisory group of Egyptian scientists and representatives from current and previous international donors such as WHO, NIAID, the European Union and the Edna McConnell Clark Foundation.

Key words: antigen - cytokine - schistosomiasis - Schistosoma - immunity - protection - resistance - vaccine

Despite two decades of widespread chemotherapy with safe and effective drugs the number of individuals with active schistosomiasis worldwide remains at about 200 million. Although animal experimentation has produced a wealth of data and there is overwhelming evidence that humans respond to schistosome infection by acquiring at least some degree of resistance, we know surprisingly little about the protective mechanisms in man. Both humoral and cell-mediated mechanisms are involved in protection but the complex regulation of immune responses does not permit a simple solution. Even if the development of a schistosomiasis vaccine requires a major research effort many factors justify making the investment needed (Table I). It appears that the immune system has

Fax: +41-22-791-4854. E-mail: bergquistn.who.ch Received 4 May 1998

Accepted 31 August 1998 been forced to balance responses associated with resistance to parasite invasion and against those that suppress the granulomatous reactions against eggs trapped in the host. The coexistence of activated macrophages, different kinds of T-cells and antibodies, both effective ones and such which block protective responses (Hagan et al. 1991, Butterworth et al. 1992), accentuate the dualistic aim of the immune system. Clinical trials would permit the study of the underlying constituents of the consolidated response in the human host and even if current vaccine candidates may not be the final answer to controlling schistosomiasis, measurable protection will be provided and safety is assured by the availability of efficacious drugs with few side effects. It should, in this context, be remembered that the pathology is directly correlated to the number of schistosome eggs in the host and a vaccine can achieve its effect both by offsetting parasite entry and development and by interfering with the production and delivery of eggs.

The rationale for schistosomiasis vaccine development can be summarized as follows: (1) since 
morbidity, rather than sterile immunity is the target, only a partially protective vaccine is required; (2) high-level protection is consistently realized with irradiated cercariae; (3) vaccines have proved an excellent means of cost-effective control of many infectious diseases; (4) rapid reinfection demands continuing treatment and drug delivery requires an infrastructure which must be both elaborate and reliable in the long term; (5) expanded chemotherapy programmes increase the risk of drug-resistance; and (6) a control approach based on chemotherapy followed by vaccination would integrate short-term effect with long-term protection.

\section{TABLE I}

Schistosomiasis vaccine development: pros and cons

\begin{tabular}{ll}
\hline Advantages & Disadvantages \\
\hline $\begin{array}{l}\text { Safe and effective drugs } \\
\text { in use }\end{array}$ & $\begin{array}{l}\text { Drug resistance } \\
\text { possible }\end{array}$ \\
$\begin{array}{l}\text { Control programmes } \\
\text { successful }\end{array}$ & $\begin{array}{l}\text { Retreatment sched- } \\
\text { ules required }\end{array}$ \\
Immunity acquired naturally & Still contended \\
$\begin{array}{l}\text { Proof of principle shown beyond } \\
\text { doubt (irradiated cercariae) }\end{array}$ & $\begin{array}{l}\text { Approach not appli- } \\
\text { cable for humans }\end{array}$ \\
$\begin{array}{l}\text { Effective vaccine candidates } \\
\text { exist to 70\% protection }\end{array}$ & $\begin{array}{l}\text { Protective mecha- } \\
\text { nisms in (up humans } \\
\text { reported }\end{array}$ \\
$\begin{array}{l}\text { largely unknown) } \\
\text { purtial immunity required }\end{array}$ & $\begin{array}{l}\text { Required level of } \\
\text { protection unknown }\end{array}$ \\
$\begin{array}{l}\text { Absence of parasite replication } \\
\text { the final (human) host }\end{array}$ & $\begin{array}{l}\text { Reinfection contri- } \\
\text { butes to in build-up } \\
\text { of worm burdens }\end{array}$ \\
Several approaches available & $\begin{array}{l}\text { Possibly difficult to } \\
\text { combine }\end{array}$ \\
\hline
\end{tabular}

The design of a successful vaccine will be based not only on the most effective way of inducing immunity but also on the technical feasibility of vaccine production. Therefore, the prospects of passage through existing regulatory bodies and the ease of incorporation into immunization delivery programmes must be part of the strategy from the very beginning. Schistosomiasis vaccines should be developed in close cooperation with the needs of current chemotherapy-based control programmes as the long-term complement to drug treatment. Drug treatment would obviously have to be continued after vaccination, albeit with longer intervals between treatment interventions than called for in current schedules.

Efforts to develop a schistosomiasis vaccine deal mainly with Schistosoma mansoni which reflects the fact that the life cycle of this species is the easiest to maintain in the laboratory. This should not, however, be interpreted as lack of interest in the other main causes of human schistosomiasis; on the contrary there is now a genuine interest in developing vaccines against $S$. japonicum (Waine et al. 1997) and S. haematobium (Trottein et al. 1992) as well.

\section{VACCINE TARGETS AND APPROACH}

Using irradiated cercarial immunization, both cell-mediated and humoral immune mechanisms can be seen to develop and contribute to protection in given model systems, ranging from rodents to non-human primates, and by manipulation of these two arms of immunity, the optimization of resistance to levels above $80 \%$ is feasible (Coulson $\&$ Wilson 1997). Even if irradiated cercariae consistently produce protection at this level, delivery problems, the need for a standardized product and safety considerations rule out this approach for human use. There are, however, several other possibilities (Table II) some of which, such as in-

TABLE II

Approaches to vaccine development

\begin{tabular}{|c|c|c|c|}
\hline $\begin{array}{l}\text { Point of } \\
\text { intervention }\end{array}$ & Targets and mechanisms & $\begin{array}{l}\text { Agents } \\
\text { identified }\end{array}$ & $\begin{array}{l}\text { Models } \\
\text { tested }\end{array}$ \\
\hline Skin penetration & Inhibition of cercarial transformation & Some & None \\
\hline $\begin{array}{l}\text { Larval growth (skin to } \\
\text { portal system) }\end{array}$ & Killing of shistosomula through membrane disruption & About 50 & About 20 \\
\hline Male/female pairing & $\begin{array}{l}\text { Interference with the presumed inter-parasite } \\
\text { signalling system }\end{array}$ & None & None \\
\hline Reproduction & Interference with egg production and delivery & Two & One \\
\hline Embryonation & Inhibition of miracidial maturation & None & None \\
\hline Granuloma formation & $\begin{array}{l}\text { Induction of specific T cell tolerance } \\
\text { Modulation or blocking of cytokine action }\end{array}$ & $\begin{array}{l}\text { Some } \\
\text { Some }\end{array}$ & $\begin{array}{l}\text { A few } \\
\text { A few }\end{array}$ \\
\hline
\end{tabular}


terfering with the inter-parasite signalling system (Haseeb \& Eveland 1991), might seem farfetched as this point, while others such as, for example, cercarial antigens (McKerrow et al. 1985) could (and should) rapidly be developed as they would complement the current focus on the schistosomulum membrane. Anti-pathology vaccination (Garcia et al. 1989, Flores-Villanueva et al. 1994, Wynn 1996) remains a distinct and attractive alternative but as vaccines geared at inducing protection against infection/reinfection have already reached the level where clinical trials can be contemplated, it would be expedient to move ahead with these candidates without delay. The ultimate vaccine might have to be a combination of two or more antigens and although attempts to combine full-length protein antigens from the same stage have not been encouraging, the possibility of synergistic action would increase by incorporating antigens from different developmental stages of the parasite.

As recombinant DNA techniques cannot yet be utilized to produce carbohydrate antigens it is more useful to focus on antigens which are predominantly of protein nature. In addition, carbohydrate antigens often cross-react with egg antigens and augment the risk of activating granulomatous reactions. The comparison between the relatively small granulomas of chronic schistosomiasis and those associated with early infection (Domingo \& Warren 1968, Von Lichtenberg 1987) and the fact that most anti-carbohydrate antibodies eventually become down-regulated (Omer-Ali et al. 1989), also emphasizes the usefulness of protein antigens. The list of published schistosome antigens, now numbering in excess of one hundred, comes from various parasite stages with the schistosomulum surface membrane being the preferential target. Characteristics which are required for further development include: (1) significant reduction of worm burdens and/or egg production compared to controls shown consistently in two different, commonly used experimental animal species; (2) demonstrated induction of human cell-mediated and humoral immune responses; (3) inclusion of a sufficient number of protective epitopes to overcome MHC restriction in hosts and genetic variation of parasite strains; (4) compatibility with approved adjuvants (if needed) and stability of vaccine formulations; (5) lack of gross side effects; (6) ease of incorporation into delivery programmes; and (7) ease of passage through regulatory authorities.

The six vaccine candidates shown in Table III were selected for further studies based on these and other criteria, the most important being that they are well-explored antigens with reported consistent high protection shown in various experi-

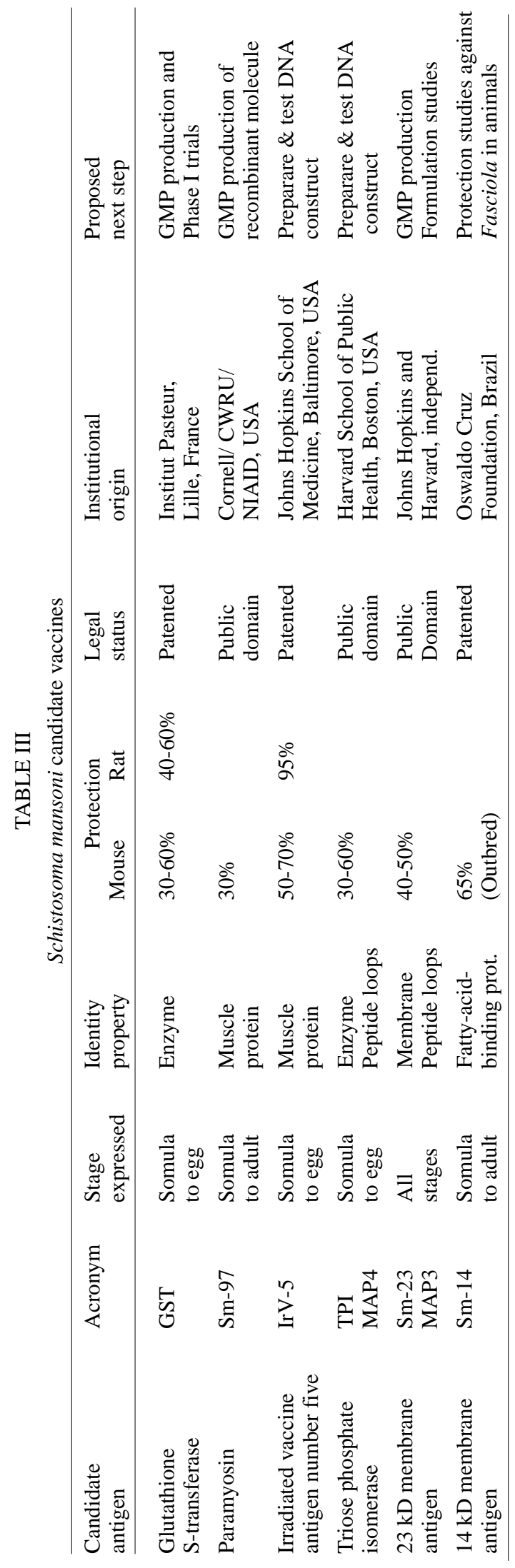


ments and laboratories. They include paramyosin (Pearce et al. 1988), IrV-5 a myosin-like molecule implicated in the irradiated cercariae model (Soisson et al. 1992), and Sm14, a protein characterized by its ability to bind fatty acids (Moser et al. 1991, Tendler et al. 1996). Glutathione S-transferase or GST (Balloul et al. 1987, Trottein et al. 1992, Capron et al. 1995) is of particular interest due to its effect on worm fecundity resulting in suppressed egg production and addition to its antiinfection activity. Triose-phosphatase isomerase (TPI) (Shoemaker et al. 1992) and Sm23 (Reynolds et al. 1992, Köster et al. 1993) represent antigens, from which "mimeotopes" of the relevant epitopes of the full-length molecule, MAP3 and MAP4, respectively, have been constructed using synthetic peptides (Reynolds et al. 1994).

\section{INDEPENDENT TESTING IN MICE}

The accumulation of results from the laboratory and the field, strongly supporting the notion of reducing morbidity due to schistosomiasis through vaccination, has begun to require the coordination of international research efforts and confirmation of reported data. Responding to this need, the UNDP/World Bank/WHO Special Programme for Research and Training in Tropical Diseases (TDR) called a series of meetings in the early 1990s which recommended the establishment of a procedure to test the reported protection results of the six priority antigens independently and to attempt correlating human immune responses to these antigens with pre- and post-treatment egg excretion records in people chronically exposed to S. mansoni. For the independent testing, two wellknown laboratories with long-term experience in experimental schistosomiasis were contracted by TDR to carry out parallel independent tests in C57BL6 and BALB/c mice for each vaccine candidate. The various groups of mice were immunized according to the specifications of the investigators providing the antigens but a standardized protocol, using the NMRI Puerto Rican strain of S. mansoni, was followed each time the challenge was performed. Each mouse received 150 cercariae percutaneously by a one-hour exposure of the tail or abdomen and all perfusions were carried out six weeks later. Unexpectedly, the consolidated results demonstrated that the stated goal of consistent induction of $40 \%$ protection or better was not reached with any of the vaccine formulations. It was felt that instability of formulations, probably accentuated by shipping under less than ideal conditions, had contributed to these unsatisfactory results and it was concluded that antigen presentation plays a more important role than previously appreciated. In spite of the disappointing results of the inde- pendent mouse tests the programme for human correlate studies in the field continues (see below). The new data expected from the human studies will contribute needed information to the selection of antigens but further independent tests in mice will have to be carried out.

\section{VACCINE DELIVERY ASPECTS}

The results described above underline the need for further exploration of antigen formulation with reference to influence and stability of adjuvants. This can be as important and influential in regard to outcome as the epitope chosen. Few adjuvants are approved for human use but various new types of adjuvants are currently under study (Cox \& Coulter 1997). However, at this time the discovery and selection of a superior adjuvant for human use must be deemed to be an empirical and lengthy process which might eventually have to involve non-human primate studies. On the other hand, swift progress on a completely novel approach, DNA vaccination, has propelled this unorthodox approach to centre stage. The injection of plasmids carrying DNA sequences capable of producing antigens in vivo bypasses a number of problems, in particular antigen purification and largescale industrial production, and promises very much of a panacea for future vaccine production. Few obstacles seem to obstruct rapid exploitation of this attractive new technology and, indeed, several DNA vaccines have already reached the stage of clinical trials in the US. Phase I clinical trials using DNA constructs of Influenza strains and the human immunodeficiency virus (HIV) are already under way and similar trials will soon start with one of the malaria vaccine candidates. Although little is understood about the so-called immunostimulatory sequences in some delivery systems, new adaptations such as, for example, the inclusion of cytokine genes, are continuously developed. Safety issues appear not to be a problem but questions on long-term DNA integration, homologue sequences and the risk of stimulating anti-DNA responses may yet require attention. Although further evaluations including studies of plasmid integration and process issues will be needed before human vaccination, various schistosome DNA constructs, including S. japonicum ones (Waine et al. 1997), have also been produced and tested in mice with encouraging results.

\section{PRECLINICAL TESTING}

Determination of antibody isotypes in clinical schistosomiasis does not present a problem but it is only during the last few years that advances in relation to the study of cytokine activation and interplay have allowed detailed investigations of human cell-mediated immunity. Relying on this 
new in vitro technology quantitative evaluation of the pattern of immunological responses triggered by each antigen was carried out in humans living in endemic areas. Of the two subsets of T helper cells governing the immune response, $\mathrm{Th}_{1}$ predominantly activates the cellular arm of the immune system through interferon-gamma (IFN-g) and interleukin two (IL-2), whilst $\mathrm{Th}_{2}$ stimulation is characterized by antibody production in association with IL-4 and IL-5, including immediate hypersensitivity ( $\operatorname{IgE})$ and eosinophilia typical for helminth infections. The role of these T-cell subpopulations in human schistosomiasis is intensely studied and it seems that IL-10 plays a major role in the overall regulation of the immune response to schistosomes, at least in infection due to $S$. mansoni (Correa-Oliveira et al. 1997). Previously, Zwingenberger et al. $(1989,1990)$ had reported a reversal of the IFN- $g$ IL-4 balance and disturbed expressions of the ratio of $\mathrm{CD} 3+$ to $\mathrm{CD} 8+$ cells which exceed three months' duration after cure in Brazilians infected with S. mansoni. In Africa, high levels of specific IgE antibodies in patients with $S$. mansoni or $S$. haematobium correlate with posttreatment resistance (Hagan et al. 1991, Dunne et al. 1995). Recent data emanating from mouse studies indicate that IL-4 is pivotal in preventing cachexia deaths in schistosomiasis via egg-induced TNF and NO-mediated gut lesions (Pearce et al. 1996). Surely, IL-4 is involved in protection mechanisms against reinfection and the strengthening of resistance by vaccination might alter the balancing mechanisms conserving the immunological equilibrium in the host.

With reference to human responses to each of the six priority antigens, protocols were designed to detect whether or not there is a statistical correlation between egg excretion records and immune responses of people chronically exposed to $S$. mansoni. In Brazil, Egypt and Kenya, well-characterized groups of about 200 individuals were selected, tested and treated and then followed-up at three months and one year afterwards. Egg counts were recorded along with levels of specific antibody isotypes and IFN-g, IL-2, IL-4, IL-5 and IL-10 responses to stimulation with the antigens under investigation. Although the detailed data analysis is not yet available, it is clear that a majority of the antigens investigated consistently elicited in vitro immunological responses compatible with protection. In Egypt, the test results in the 225 patients investigated could also be analysed retrospectively as detailed previous data were available for a majority of the subjects permitting them to be classified as putatively "resistant" or "susceptible". The emerging patterns are complex and although this extensive set of data will require fur- ther in-depth analysis, i.e. taking into account the different time points and the ages of the patients, useful initial comparisons of magnitude and prevalence of responses to each vaccine candidate are provided. In addition, the data allow an evaluation of the degree of genetic restriction likely to be encountered with the various vaccine candidates. In Kenya, preliminary data resulting from the pretreatment studies, performed using crude antigenic preparations and some of the vaccine candidates, showed some high readings, especially of IL-5 when the cells were stimulated with crude worm antigens. However, due to a drought at the testing site last year and the ensuing changes in transmission patterns, it is unlikely that post-treatment reinfection studies will be feasible. In agreement with the Kenyan and Egyptian results, the strongest responses observed in Brazil were for the most part IL-5 and IFN-g and there were indications that some cytokines are more likely to be induced by certain vaccine candidates, for example IL-5 in response to paramyosin and IFN-g in response to IrV-5 and Sm14. The overallimpression is that these preliminary results in all three endemic areas support the importance of the response patterns of IFN-g/IL-5. In addition, the IgE/IgA isotypes appear to be of particular interest.

Four of the priority molecules are in the public domain, whilst two, GST and Sm14, have been patented. The promising results of these two molecules have provided an impetus for industrial interest and led to rapid progress. Due to its activity against a shared epitope between $S$. mansoni and Fasciola hepatica, Sm14 is currently under study by ISL, an Australian pharmaceutical company for vaccination against Fasciola in farm animals, while an European company is interested to develop a GST-based vaccine against $S$. haematobium. The anti-fecundity effect of GST is more pronounced in this species than in S. mansoni and morbidity due to urinary schistosomiasis is already common at the level of a few eggs per $10 \mathrm{ml}$ urine and develops rapidly into serious pathology, including bladder cancer. In addition, there is evidence that a vaccine against $S$. haematobium would be active against $S$. mansoni as well (Boulanger et al. 1995). A GMP formulation of Sh GST will be ready for clinical Phase I trials in December 1997 and, provided initial trials are successful, subsequent Phase II trials are scheduled for Niger $(S$. haematobium), Senegal (S. mansoni and $S$. haematobium) and Madagascar (S. mansoni).

\section{CONCLUSIONS AND RECOMMENDED NEXT STEPS}

The time has come to extend our knowledge about the immunological response to the vaccine candidates and their capability to protect against 
schistosome infection through controlled clinical trials. Ethical considerations would be satisfied by the provision of protection and the assurance of safety through effective drugs with few side effects. As chronic schistosomiasis must be expected to be common in any study area the potential of exacerbation is, however, an issue to take into account. Although we shall need to continue learning from animal models, in particular in relation to peptide/protein candidates and schistosome-related DNA vaccines, further progress will require shifting the focus to large-scale production and Phase I trial design. Many critical questions can only be answered by safety-first, well controlled clinical trials and it is now appropriate to move in that direction.

Building on the results from, and capacity developed in, Brazil, Egypt and Kenya, an alliance for research coordination and support dedicated to promoting vaccine candidates could achieve tangible results already in the short term. Such a research alliance is about to be formed as USAID, Cairo has pledged financial support with the backing of the US National Institute of Allergy and Infectious Diseases (NIAID), the Commission of European Communities of the European Union (CEC), and TDR. Vaccine candidates will be moved forward on two fronts, DNA-based evaluations and protein-based development. At a meeting in May 1997 in Cairo, Egypt, it was agreed to promote at least two $S$. mansoni vaccine candidate through Phase I clinical trials, primarily in Egypt and the US. A highly focused approach will be adopted scaling up the production of selected vaccine candidates under GMP and evaluating them in appropriately controlled clinical trials. Some vaccine candidates are already scheduled for clinical (GST) or veterinary (Sm14) trials and various roads are open for the other four. All vaccine candidates induce observable and fairly consistent patterns of cytokine and isotype responses and based on how well they fit the conditions in table 5, paramyosin and MAP4 (originating from TPI) seem suited for immediate GMP production and Phase I clinical trials whilst others, IrV5 and Sm23 due to production and formulation concerns, may be better suited for further development into DNA vaccines. Thus, at this time $\operatorname{IrV} 5$ and $\operatorname{Sm} 23$ will be further evaluated in animal models as DNA vaccines, whilst paramyosin and MAP4/TPI offer the greatest potential for the rapid preclinical development of a protein-based vaccine.

\section{REFERENCES}

Balloul JM, Grzych JM, Pierce RJ, Capron A 1987. A purified 28000 Dalton protein from Schistosoma mansoni adult worms protects rats and mice against experimental schistosomiasis. J Immunol 138: 34483453.

Boulanger D, Warter A, Trottein F, Mauny F, Bremond P, Audibert F, Couret D, Kadri S, Godin C, Sellin E, Capron A 1995. Vaccination of Patas monkeys experimentally infected with Schistosoma haematobium using recombinant glutathione S-transferase cloned from Schistosoma mansoni. Parasite Immunol 17: 361-369.

Butterworth AE, Dunne DW, Fulford AJ, Thorne KJ, Gachuhi K, Ouma JH, Sturrock RF 1992. Human immunity to Schistosoma mansoni: observations on mechanisms and implications for control. Immunol Invest 21: 391-407.

Capron A, Riveau G, Grzych JM, Boulanger D, Capron M, Pierce R 1995. Development of a vaccine strategy against human and bovine schistosomiasis. Background and update. Mem Inst Oswaldo Cruz 90: 235-240.

Coulson PS, Wilson RA 1997. Recruitment of lymphocytes to the lung through vaccination enhances the immunity of mice exposed to irradiated schistosomes. Infect Immun 65: 42-48.

Cox JC, Coulter AR 1997. Adjuvants - a classification and review of their modes of action. Vaccine 15 : 248-256.

Correa-Oliveira R, Malaquias LCC, Falcão PL, Viana IRC, Bahia-Oliveira LMG, Silveira AMS, Fraga LAO, Prata S, Coffman RL, Lambertucci JR, CunjaMelo JR, Martins-Filho OA, Wilson RA, Gazzinelli G 1997. Cytokines as determinants of resistance and pathology in human Schistosoma mansoni infection. Brazil J Med Biol Res 31: 171-177.

Domingo EO, Warren KS 1968. Endogenous desensitization. Changing host granulomatous response to schistosome eggs at different stages of infection with Schistosoma mansoni. Am J Pathol 52: 369-372.

Dunne DW, Hagan P, Abath FG 1995. Prospects for immunological control of schistosomiasis. Lancet 345: 1488-1491.

Flores-Villanueva PO, Harris TS, Ricklan DE, Stadecker MJ 1994. Macrophages from schistosomal egg granuloma induce unresponsiveness in specific cloned Th-1 lympocytes in vitro and down-regulate schistosomal granulomatous disease in vivo. J Immunol 152: 1847-1855.

Garcia EG, Rivera PT, Mitchell GF, Evardome RR, Almonte RE, Tiu WU 1989. Effects of induction of anti-embryonation immunity on liver granulomas, spleen weight and portal pressure in mice infected with S. japonicum. Acta Tropica 46: 93-99.

Hagan P, Blumenthal UJ, Dunn D, Simpson AJ, Wilkins HA 1991. Human IgE, IgG4 and resistance to reinfection with Schistosoma haematobium. Nature 349: 243-245.

Haseeb MA, Eveland LK 1991. Schistosoma mansoni: a chemoattractive factor released by males and its receptor in females. Experentia 47: 970-974.

Köster B, Hall MRT, Strand M 1993. Schistosoma mansoni: immunoreactivity of human sera with the surface antigen Sm23. Exp Parasitol 77: 282-294.

McKerrow JH, Jones P, Sage H, Pino-Heiss S 1985. 
Purification and characterization of an elastinolytic proteinase secreted by cercariae of Schistosoma mansoni. J Biol Chem 260: 3703-3704.

Moser D, Tendler M, Griffiths G, Klinkert M-Q 1991. A $14-\mathrm{kDa} S$. mansoni polypeptide is homologous to a gene family of fatty acid binding proteins. $\mathrm{J}$ Biol Chem 266: 8447-8454.

Omer-Ali P, Mansour M, Woody JN, Smithers SR, Simpson AJG 1989. Antibody to carbohydrate and polypeptide epitopes on the surface of Schistosoma mansoni in Egyptian patients with acute and chronic schistosomiasis. Parasitology 98: 417-424.

Pearce EJ, James SL, Hieny S, Lanar DE, Sher A 1988. Induction of protective immunity against Schistosoma mansoni by vaccination with schistosome paramyosin (Sm97), a nonsurface parasite antigen. Proc Natl Acad Sci USA 85: 5678-5682.

Pearce EJ,Vasconcelos JP, Brunet LR, Sabin EA 1996 IL-4 in schistosomiasis. Exp Parasitol 84: 295-299.

Reynolds SR, Dahl CE, Harn D 1994. T and B epitope determination and analysis of multiple antigenic peptides for the $S$. mansoni experimental vaccine triosephosphate isomerase. J Immunol 152: 193-200.

Reynolds SR, Shoemaker CB, Harn DA 1992. T and B cell epitope mapping of Sm23, an integral membrane protein S. mansoni. J Immunol 149: 3995-4001.

Shoemaker C, Gross A, Gebremichael A, Harn D 1992. cDNA cloning and functional expression of the Schistosoma mansoni protective antigen triose-phosphate isomerase. Proc Natl Acad Sci USA 89: 1842-1846.

Soisson LM, Masterson CP, Tom TD, McNally MT, Lowell GH, Strand M 1992. Induction of protective immunity in mice using a 62-kDa recombinant frag- ment of a Schistosoma mansoni surface antigen. $J$ Immunol 149: 3612-3620.

Trottein F, Godin C, Pierce RJ, Sellin B, Taylor MG, Gorillot I, Silva MS, Lecocq JP, Capron A 1992. Inter-species variationof schistosome $28 \mathrm{kDa}$ glutathione S-transferase. Mol Biochem Parasitol 54: 63-72.

Tendler M, Brito CA, Vilar MM, Serra-Freire N, Diogo CM, Almeida MS, Delbem AC, Da Silva JF, Savino W, Garratt RC, Katz N, Simpson AS 1996. A Schistosoma mansoni fatty-acid-binding protein, Sm14, is the potential basis of a dual-purpose anti-helminth vaccine. Proc Natl Acad Sci USA 93: 269-273.

Von Lichtenberg F 1987. Consequences of infection with schistosomes, p. 185-232. In D Rollinson, AJG Simpson (eds), The Biology of Schistosomes, Academic Press, London and New York.

Waine GJ, Yang Wen, Scott JC, McManus DP, Kalinna BH 1997. DNA-based vaccination using Schistosoma japonicum (Asian blood-fluke) genes. Vaccine 15: 846-848.

Wynn TA 1996. Development of an antipathology vaccine for schistosomiasis. Ann N Y Acad Sci 797: 191-195.

Zwingenberger K, Harms G, Vergetti de Siqueira JG, Correia-dacal AR, Janssen-Rosseck R, Bienzle U, Feldmeier H 1989. T-cell phenotype alterations in hepatosplenic schistosomiasis mansoni normalize after chemotherapy. Immunobiol 179: 342-352.

Zwingenberger K, Richter J, Taupitz S, Siqueira-Vergetti JG, Correia-Dacal AR 1990. Altered generation of interleukin 1 in chronic human schistosomiasis mansoni. Scand J Immunol 31: 729-736. 
